\title{
Working Memory and the Organization of Brain Systems
}

\author{
Yael Shrager, ${ }^{1}$ Daniel A. Levy, ${ }^{2}$ Ramona 0. Hopkins, ${ }^{3}$ and Larry R. Squire ${ }^{4}$ \\ ${ }^{1}$ University of California, San Diego, La Jolla, California 92093, ${ }^{2}$ Weizmann Institute of Science, Rechovot 76100, Israel, ${ }^{3}$ Brigham Young University, Provo, \\ Utah 84602, and ${ }^{4}$ Veterans Affairs Healthcare System, San Diego, California 92161
}

\begin{abstract}
Working memory has historically been viewed as an active maintenance process that is independent of long-term memory and independent of the medial temporal lobe. However, impaired performance across brief time intervals has sometimes been described in amnesic patients with medial temporal lobe damage. These findings raise a fundamental question about how to know when performance depends on working memory and when the capacity for working memory has been exceeded and performance depends on long-term memory. We describe a method for identifying working memory independently of patient performance. We compared patients with medial temporal lobe damage to controls who were given either distraction or no distraction between study and test. In four experiments, we found concordance between the performance of patients and the effect of distraction on controls. The patients were impaired on tasks in which distraction had minimal effect on control performance, and the patients were intact on tasks in which distraction disrupted control performance. We suggest that the patients were impaired when the task minimally depended on working memory (and instead depended substantially on long-term memory), and they performed well when the task depended substantially on working memory. These findings support the conclusion that working memory (active maintenance) is intact after medial temporal lobe damage.
\end{abstract}

Key words: amnesia; hippocampal function; hippocampus; memory; working memory; long-term memory

\section{Introduction}

Working memory is a fundamental concept in cognitive neuroscience and psychology, and in these disciplines it has largely replaced the less precise term, short-term memory (Baddeley, 2003). Working memory refers to the capacity to maintain temporarily a limited amount of information in mind, which can then be used to support various cognitive abilities, including learning and reasoning (Baddeley and Hitch, 1974). Historically, working memory (or short-term memory) was distinguished from long-term memory (a large-capacity, stable storage system), and for the past half century this distinction has been fundamental to understanding how the brain has organized its memory functions (Waugh and Norman, 1965; Baddeley and Warrington, 1970). For example, early studies of amnesic patients with medial temporal lobe damage found working memory to be intact despite markedly impaired performance on tasks of longterm memory (Drachman and Arbit, 1966; Milner, 1972). Indeed, in psychological science, one finds the suggestion that what is spared in amnesia provides the best evidence for the construct of working memory as well as a good definition of it (Atkinson and Shiffrin, 1968; Pashler and Carrier, 1996). The view has been that working memory is independent of the hippocampus and other medial temporal lobe structures, whereas these structures are essential for the formation of long-term memory (Milner, 1972).

\footnotetext{
Received Jan. 17, 2008; revised March 14, 2008; accepted March 28, 2008

This work was supported by the Medical Research Service of the Department of Veterans Affairs, National Institute of Mental Health Grant MH24600, the Metropolitan Life Foundation, and a National Science Foundation Predoctoral Fellowship (Y.S.). We thank Jennifer Frascino and Mark Starr for assistance.

Correspondence should be addressed to Larry R. Squire at the above address. E-mail: Isquire@ucsd.edu. DOI:10.1523/JNEUROSCI.0710-08.2008

Copyright $\odot 2008$ Society for Neuroscience $\quad 0270-6474 / 08 / 284818-05 \$ 15.00 / 0$
}

These ideas have been challenged recently by the proposal that working memory might sometimes depend on medial temporal lobe structures. Specifically, patients with medial temporal lobe damage were found to be impaired at remembering information across brief time intervals (Hannula et al., 2006; Nichols et al., 2006; Olson et al., 2006a,b; Hartley et al., 2007). On the one hand, these impairments would seem to require a revision of a longstanding principle of brain organization. On the other hand, the impairments might have occurred because the capacity for working memory was exceeded in these cases [also see discussion in Hannula et al. (2006) and Nichols et al. (2006)]. In fact, there is circularity in the way that working memory is traditionally understood (i.e., working memory has been characterized as the kind of memory that is spared in amnesia, but amnesia is also thought to be a condition in which working memory is intact). What is needed is a method for identifying and measuring working memory that is entirely independent of the performance of amnesic patients.

We have measured the contribution of working memory to normal performance by introducing distraction between study and test to interrupt the active maintenance of studied information. We reasoned as follows: if amnesic patients perform well on tasks when they can operate within working memory capacity (that is, by active maintenance), then controls given the same tasks should be impaired by distraction between study and test, because distraction would disrupt the active maintenance process. Conversely, if amnesic patients perform poorly when their working memory capacity is exceeded, then controls given the same tasks should be minimally affected by distraction between study and test (because performance is now supported more by long-term memory than by active maintenance). 


\section{Materials and Methods}

Participants. Eight patients participated. Two patients (E.P. and G.P., aged 83 and 60, respectively) have severe memory impairment resulting from viral encephalitis, together with intact perceptual and intellectual functions (Bayley et al., 2006; Shrager et al., 2006). These patients have demonstrated virtually no new learning since the onset of their amnesia, and during repeated testing over many weeks they do not recognize that they have been tested before (Bayley et al., 2005a). Estimates of medial temporal lobe damage were based on quantitative analysis of magnetic resonance (MR) images and data from four controls for each patient. E.P. and G.P. have average bilateral reductions in hippocampal volume of 97 and $96 \%$, respectively. The volume of the parahippocampal gyrus (temporopolar, perirhinal, entorhinal, and parahippocampal cortices) is reduced by 94 and $93 \%$, respectively.

Six patients have damage thought to be limited to the hippocampus and are moderately amnesic. R.S. and J.R.W. participated only in the test of relational information. A.B. participated only in the faces test. A.B. and J.R.W. (aged 66 and 43, respectively) became amnesic after cardiac arrest. G.W. and R.S. (aged 47 and 50, respectively) became amnesic after drug overdoses and associated respiratory failure. K.E. (aged 65) became amnesic after an episode of ischemia associated with kidney failure and toxic shock syndrome. L.J. (the only female; aged 68) became amnesic during a 6 month period in 1988 with no known precipitating event. Her memory impairment has remained stable since that time. Estimates of medial temporal lobe damage were based on quantitative analysis of MR images. K.E., L.J., R.S., G.W., and J.R.W. have average bilateral reductions in hippocampal volume of $49,46,33,48$, and $44 \%$, respectively (Bayley et al., 2005b; Gold and Squire, 2005). The volume of the parahippocampal gyrus is reduced by $17,-8,1,12$, and $6 \%$, respectively (all values within two SDs of the control mean). A.B. was unable to participate in MR imaging.

Nine coronal MR images for seven of the patients (all but A.B.), together with detailed descriptions of the lesions, are presented as supplemental material (available at www.jneurosci.org).

Recognition memory for names and faces: patients versus controls. The 2 patients with large medial temporal lobe lesions, 4 patients with hippocampal lesions, and 12 age- and education-matched controls were tested for their memory for names and faces. In the names task (only three of the hippocampal patients participated), three surnames were presented one at a time for $1 \mathrm{~s}$ each. After an unfilled delay of $14 \mathrm{~s}$, memory was tested with a single probe stimulus that asked participants to decide whether a name was the same as or different from one of the studied names ( 8 trials per block, 64 total trials). In the faces task, a single face was presented for $1 \mathrm{~s}$. After a delay of 2,7 , or $14 \mathrm{~s}$, memory was tested with a single probe stimulus that asked participants to decide whether a face was the same as or different from a studied face ( 2 sessions, 4 blocks per delay in each session presented in pseudorandom order, 8 trials per block, 64 total trials at each delay). The interval between trials was selfpaced in all experiments reported here.

Recognition memory for names and faces: effect of distraction on controls. Twelve age- and education-matched controls took the names and the faces tests, and on half the trials were distracted during seconds $4-7$ of the delay. For the distraction, participants counted a series of $10-20$ rapidly presented names (in the names condition, each name was presented for $250 \mathrm{~ms}$ ) or 10-20 faces (in the faces condition, each face was presented for $250 \mathrm{~ms}$ ) and reported their count at the end of the delay ( 2 blocks per condition, 8 trials per block, 16 total trials for names and 16 for faces). The no-distraction condition also consisted of 16 trials for names and 16 for faces. The eight blocks (two each for no distraction and distraction in the names condition, and two each in the faces condition) were presented in pseudorandom order.

In designing an appropriate distraction task that will effectively disrupt active maintenance, one can begin by using stimuli of the same type as the studied stimuli. Yet another issue of potential importance is to engage participants in the same domain of working memory (e.g., phonological loop vs visual sketchpad) that is engaged during study. Because this second criterion might not have been met in our first distraction condition with faces (because participants may have focused on counting the faces, not processing them as faces), we performed a second distraction condition (faces only), designed to be more relevant to the processing of faces. Ten of the same 12 controls viewed a series of 10-20 rapidly presented faces during seconds 4-7 of the delay. On half the distraction trials, one of the faces was Bill Clinton (never presented among the first or last three images). Participants indicated at the end of each delay whether they had seen Bill Clinton (2 blocks, 8 trials/block, 16 trials total). The no-distraction condition with faces also consisted of 16 trials ( 2 blocks, 8 trials/block). The four blocks were presented in pseudorandom order.

Recognition memory for object locations: patients versus controls. The 2 patients with large medial temporal lobe lesions, 5 patients with hippocampal lesions, and 12 age- and education-matched controls were tested for object location memory. On each trial, participants studied drawings of one, two, three, four, or six colored objects (Olson et al., 2006a), each presented in one cell of a $3 \times 3$ grid. Objects were presented one at a time for $1 \mathrm{~s}$ each, and no cell was used more than once within a trial. After a delay of 1 or $8 \mathrm{~s}$, memory was tested with a single probe stimulus.

Because the probe stimulus for the recognition test always needed to be a combination of one of the studied objects and one of the studied locations, it was not possible to test recognition memory in the one-object condition (i.e., the correct answer would always be "same"). Thus, on trials where only one object was studied, recall of the object and its location was tested. Participants were shown an empty grid and asked to report what object had been presented and in which cell of the grid it had appeared.

On all other trials, one of the studied objects appeared in one of the studied locations, and participants indicated whether the object was in the same location as during study or in a different location. The one-, two-, and three-object conditions were tested in the first of two sessions, and the four- and six-object conditions were tested in the second session (in each case, 2 blocks per object condition were presented in a pseudorandom order, 8 trials per delay condition in each block, 16 total trials at each delay in each object condition).

Recognition memory for object locations: effect of distraction on controls. Eighteen age- and education-matched controls took two tests, a threeobject condition and a six-object condition. On half the trials, they were distracted during seconds $4-7$ of the delay. For the distraction, participants counted a series of 10-20 rapidly presented black-and-white objects (Snodgrass and Vanderwart, 1980) and reported their count at the end of the delay ( 2 blocks per condition, 8 trials per block, 16 trials for the three-object condition and 16 trials for the six-object condition). The no-distraction condition also consisted of 16 trials for each condition. The eight blocks (two each for no distraction and distraction in the three-object condition, and two each in the six-object condition) were presented in pseudorandom order.

\section{Results}

\section{Recognition memory for names and faces}

Patients with medial temporal lobe damage and matched controls tried to remember either three names or a single face for short time intervals (for details, see Materials and Methods). The patients performed as well as controls when they tried to remember three names for $14 \mathrm{~s}$ (patients, 94.4\% correct; controls, $94.5 \%$ correct) (Fig. 1a). For faces, performance was good after $2 \mathrm{~s}$ (patients, 99.1\% correct; controls, 98.8\% correct) and also $7 \mathrm{~s}$ (patients, $96.6 \%$ correct; controls, $97.8 \%$ correct). In contrast, the patients were impaired when asked to remember a single face for $14 \mathrm{~s}\left(93.2\right.$ vs $98.0 \%$ correct; $\left.t_{(16)}=2.6 ; p<0.03\right)$ (Fig. $\left.1 a\right)$. Average proportion correct, hit, and false alarm scores for each group, together with corresponding SEs, are presented for all experiments in supplemental tables (available at www. jneurosci.org as supplemental material). All results in all the experiments reported here followed the same pattern when analyses were based either on $d^{\prime}$ scores or on arcsine transformations of the percentage correct scores [with the standard correction for $100 \%$ scores: $1-1 /(4 n)$, where $n=$ number of 

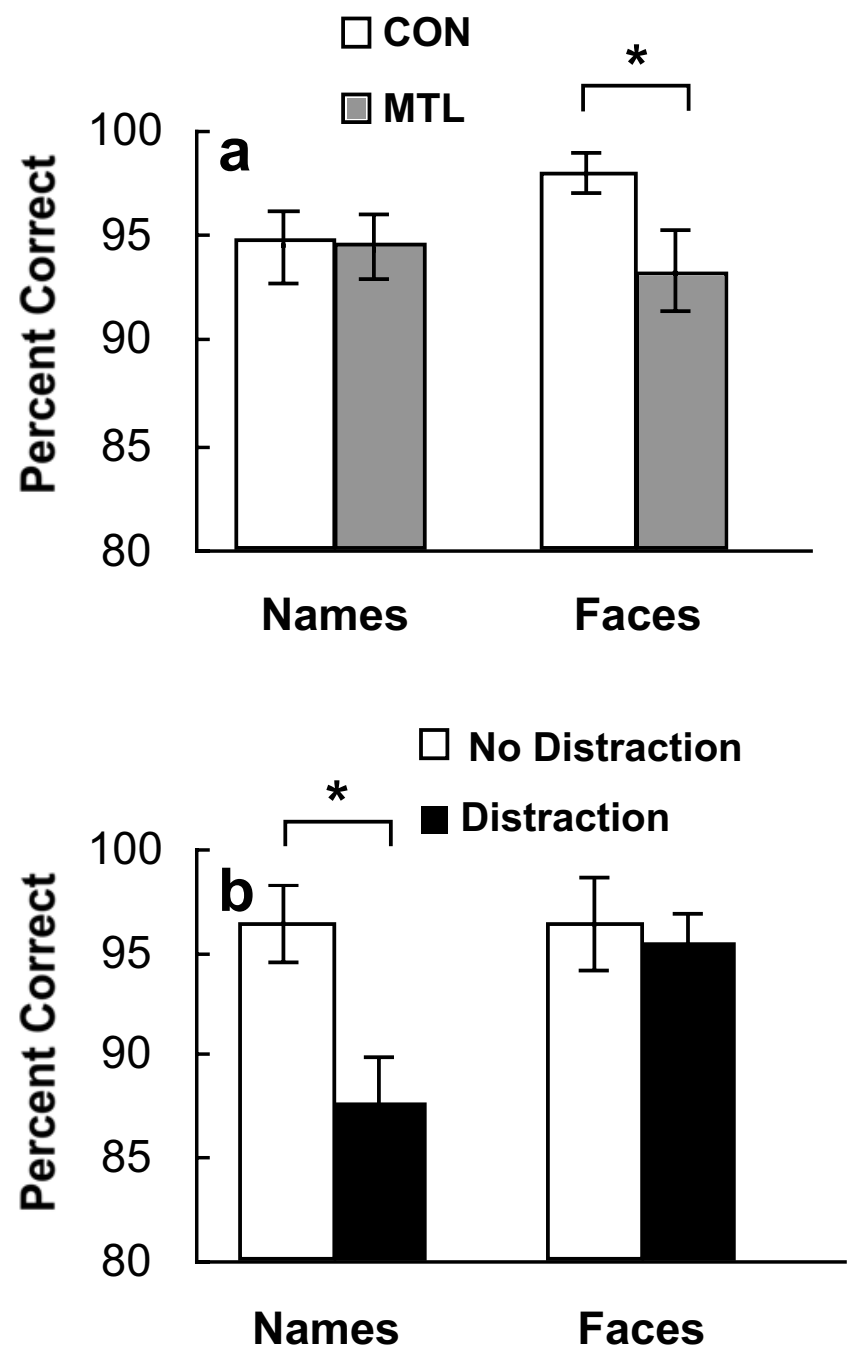

Figure 1. Memory for names and faces. $\boldsymbol{a}$, Twelve controls ( $(\mathrm{CON})$ and patients with medial temporal lobe damage (MTL) tried to remember three names for $14 \mathrm{~s}$ ( $n=5$ patients) or a single face for $14 \mathrm{~s}$ ( $n=6$ patients). $\boldsymbol{b}$, Twelve controls were tested in both the names condition and the faces condition with and without a distraction task during the $14 \mathrm{~s}$ delay. Error bars indicate SE. Asterisks indicate $p<0.05$.

trials]. Arcsine transformations were used because the scores sometimes deviated from a normal distribution.

The question of interest is whether the impairment found in the faces condition at the $14 \mathrm{~s}$ delay reflects impaired working memory or impaired long-term memory. To distinguish between these possibilities, we tested the effect of distraction between study and test on control performance in both the faces condition and the names condition at a $14 \mathrm{~s}$ delay. Figure $1 b$ shows that in the names condition, performance was affected by distraction (96.4 vs $87.5 \%$ correct; $t_{(11)}=4.2 ; p=0.001$ ). In contrast, distraction had no effect in the faces condition $(96.4 \%$ correct for the no-distraction condition vs $95.3 \%$ correct for the distraction condition). Performance on the distracter (counting) tasks was comparable in the two conditions (average count error $=2.8$ and 2.5 , respectively; $\left.t_{(11)}=1.4 ; p=0.19\right)$. An additional distracter task in the faces experiment used a task more relevant to the processing of faces (see Materials and Methods). Again, distraction had no effect in the faces condition (no distraction $=97.5 \%$ correct vs distraction $=98.1 \%$ correct) .

These results reveal a correspondence between the perfor- mance of amnesic patients and the effects of distraction on controls. Distraction impaired controls on the names test, presumably because the distraction interfered with an active maintenance process based on rehearsal. Distraction did not affect performance on the faces test, presumably because the information is difficult to maintain actively (rehearse) and must depend on long-term memory shortly after the information is presented [for a similar interpretation of face memory, see Warrington and Taylor (1973)]. We suggest that amnesic patients were intact when the task was supported by rehearsal (working memory for names) but were impaired in the case of faces when rehearsal was less effective and performance had to depend on long-term memory.

\section{Recognition memory for object locations}

It has been suggested that medial temporal lobe structures, particularly the hippocampus, are important for relational memory (Cohen et al., 1999). In the strong version of this view, for tasks involving relational information, medial temporal lobe structures are needed not only for long-term memory but also for working memory (Hannula et al., 2006; Olson et al., 2006a). We tested this idea following the same logic as in the names and faces test. Patients and controls tried to remember one, two, three, four, or six object locations for delays of 1 or $8 \mathrm{~s}$. The patients performed as well as controls in all five object conditions at the $1 \mathrm{~s}$ delay (controls $=90.1 \%$ correct; patients $=90.5 \%$ correct $)$ and in four of the five conditions at the $8 \mathrm{~s}$ delay (one, two, three, and four objects; controls $=93.3 \%$ correct; patients $=91.3 \%$ correct). Group means are shown for each individual condition in supplemental tables (available at www.jneurosci.org as supplemental material). The patients were impaired only when trying to remember six object locations over an $8 \mathrm{~s}$ delay (controls $=$ $71.9 \%$ correct; patients $=60.7 \%$ correct; $\left.t_{(17)}=2.7 ; p<0.02\right)$. Figure $2 a$ shows the good performance of the patients when they remembered three objects across $8 \mathrm{~s}$ and their poor performance when they tried to remember six objects.

The question of interest is whether the impairment found in the six-object condition at the $8 \mathrm{~s}$ delay reflects impaired working memory or impaired long-term memory. We hypothesized that the performance of patients in the three-object condition at $8 \mathrm{~s}$ was intact because performance in this condition relied on an active maintenance process. Further, we hypothesized that the performance of patients in the six-object condition at $8 \mathrm{~s}$ was impaired because now performance relied, at least in part, on long-term memory. Accordingly, following the same logic as in the names and faces tests, we expected distraction to disrupt control performance in the three-object condition and to disrupt performance much less in the six-object condition.

To test these predictions, we next tested the effect of distraction between study and test on control performance in both the three-object condition and the six-object condition at an $8 \mathrm{~s}$ delay. Figure $2 b$ shows that in the three-object condition, performance was markedly affected by distraction $(91.3$ vs $67.4 \%$ correct; paired $\left.t_{(17)}=6.6 ; p<0.001\right)$. Distraction also affected performance in the six-object condition $(73.6$ vs $65.6 \%$ correct; paired $\left.t_{(17)}=2.2 ; p<0.05\right)$, albeit much less than in the threeobject condition. Importantly, there was a number of objects $\times$ distraction interaction $\left(F_{(1,17)}=6.8 ; p<0.02\right)$, indicating that the effect of distraction was greater in the three-object condition than in the six-object condition. It is also important that there was no floor effect in the two distraction conditions. Thus, all group means were above chance (50\%; $p$ values $<0.05$ ), and $50 \%$ was well outside of the $95 \%$ confidence interval for the scores in 

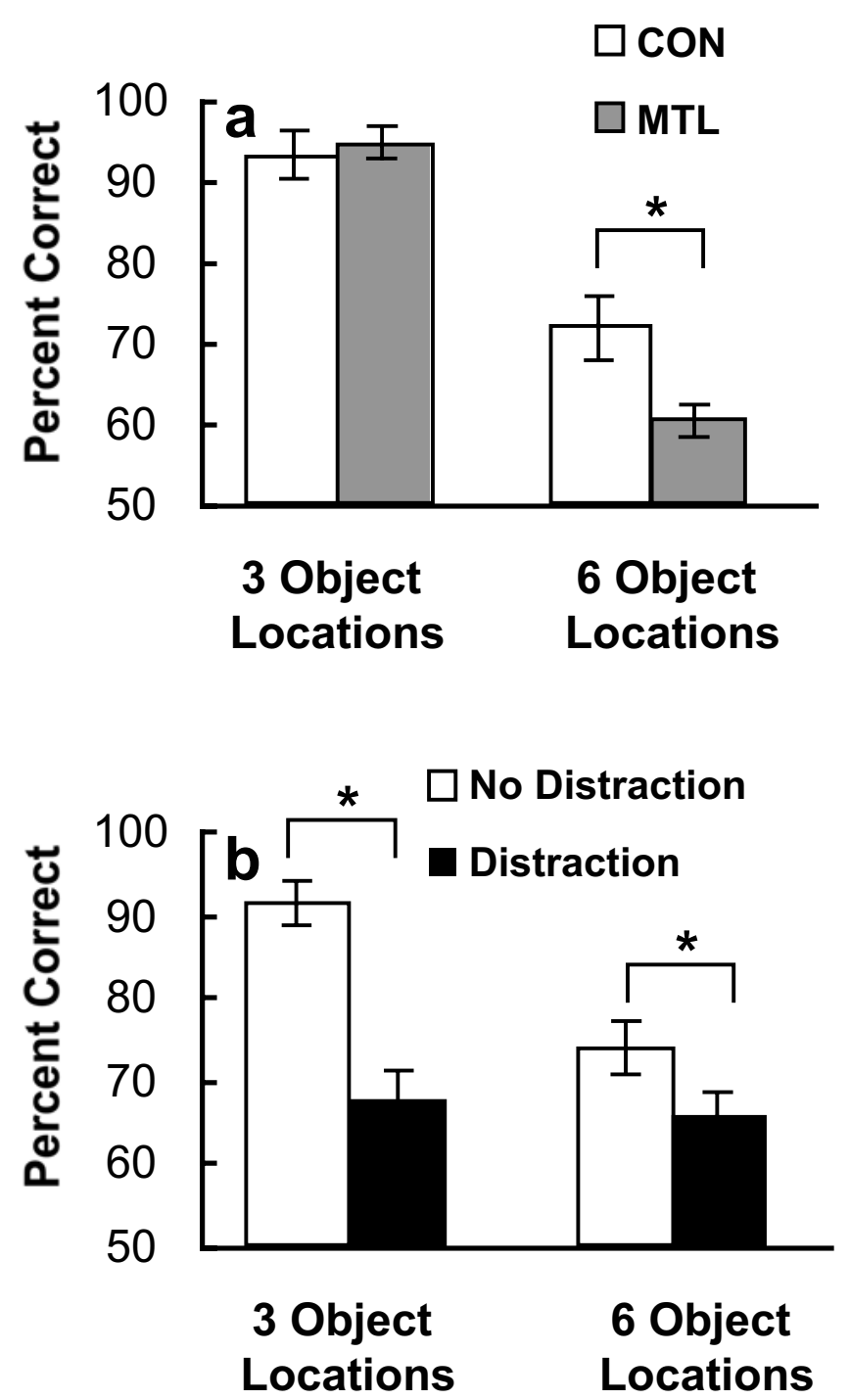

Figure 2. Memory for object locations. $\boldsymbol{a}$, Twelve controls (CON) and seven patients with medial temporal lobe damage (MTL) tried to remember three object locations or six object locations for $8 \mathrm{~s}$ (16 trials per condition). $\boldsymbol{b}$, Eighteen controls tried to remember three or six object locations with or without a distraction task during the delay (16 trials per condition, 64 trials total). Error bars indicate SE. Asterisks indicate $p<0.05$.

each condition (for the three-object distraction condition, $67.4 \pm$ $7.4 \%$; for the six-object distraction condition, $65.6 \pm 6.1 \%$ ). Performance on the distracter (counting) task was comparable for the three-object and six-object conditions (average count error $=$ 2.2 and 2.3 , respectively; $\left.t_{(17)}=0.4 ; p=0.68\right)$.

Still another way to consider the data from the distraction experiment with object locations is to look at the benefit afforded by the use of working memory. That is, one can consider the distraction conditions as a baseline, showing how controls perform when they would have difficulty using working memory. Then, one can ask how performance improves when controls are allowed to use working memory. In the three-object condition, the benefit is quite large (improvement from 67.4 to $91.3 \%$ ), whereas in the six-object condition, the benefit is minimal (improvement from 65.6 to $73.6 \%$ ). We interpret this pattern of results to mean that performance in the three-object condition depended substantially on working memory but that in the sixobject condition, performance depended mainly on long-term memory.
As with names and faces, these results reveal a correspondence between the performance of amnesic patients and the effects of distraction on controls. Distraction impaired controls to a greater degree in the three-object condition than in the six-object condition. Presumably, performance in the three-object condition depended substantially on rehearsal, and distraction interfered with active maintenance of what had been presented. In contrast, distraction had only a modest effect on the six-object condition, because what was presented exceeded working memory capacity, and performance relied substantially on long-term memory. The important finding was that distraction impaired performance in the three-object condition significantly more than in the sixobject condition.

We suggest that amnesic patients were intact in the threeobject condition because the task was supported primarily by working memory (rehearsal), and they were impaired in the sixobject condition because, although working memory likely contributed to some extent, rehearsal was not sufficient to support good performance.

\section{Discussion}

In four different tasks (memory for names, faces, three object locations, and six object locations), we found concordance between the performance of patients with medial temporal lobe damage and the effect of distraction on controls. The patients were intact on tasks in which distraction disrupted control performance, and the patients were impaired on tasks in which distraction minimally affected control performance. These findings suggest that an active maintenance process (working memory) contributed substantially to control performance when patients performed well and less so or not at all when patients were impaired. These results suggest that the active maintenance process is intact after medial temporal lobe damage. It is true that patients with medial temporal lobe damage can be impaired at remembering some kinds of stimuli after quite brief delays, even when no stimuli intervene between study and test (Hannula et al., 2006; Nichols et al., 2006; Olson et al., 2006a,b; Hartley et al., 2007; Ezzyat and Olson, 2008). However, we suggest that these findings reflect an early dependence on long-term memory, not an impairment in working memory. Working memory is limited by its low capacity and by the ease with which information can be actively maintained through rehearsal. The length of the study-test interval is not the important factor.

Our findings also address the suggestion that relational memory is critically dependent on the medial temporal lobe, regardless of whether performance depends on long-term memory or working memory. The strong version of this view holds that patients with medial temporal lobe damage should be impaired in maintaining relational information even over short delays (Olson et al., 2006a). Contrary to this idea, we found that patients with hippocampal damage, and even the two patients with extensive medial temporal lobe damage, successfully maintained up to six object-location associations (relational information) for $1 \mathrm{~s}$ and up to four object-location associations for $8 \mathrm{~s}$. We suggest that relational information can be maintained as long as the material is amenable to rehearsal and does not exceed the capacity of working memory.

Despite similarity in the tasks, the performance of our patients differed in some respects from the performance of patients in previous studies (Nichols et al., 2006; Olson et al., 2006a,b). In the faces test, our patients performed as well as controls at the $2 \mathrm{~s}$ and $7 \mathrm{~s}$ delays and were impaired only at the 14 s delay. In a previous study (Nichols et al., 2006), patients were impaired at the 7 s delay 
and performed numerically worse than our patients at that delay. In another study of memory for faces, patients were impaired when trying to remember a single face for $4 \mathrm{~s}$ (Olson et al., 2006b). In the object location test, our patients performed well at remembering up to six object locations for $1 \mathrm{~s}$ and up to four object locations for $8 \mathrm{~s}$. In a previous study (Olson et al., 2006a), patients were impaired when remembering three object locations for $1 \mathrm{~s}$ (in one of two experiments) and for $8 \mathrm{~s}$ (in two of two experiments).

Differences between patient groups might account for these differences in severity of impairment. The damage in our patients was measured using quantitative volumetric analysis of MR images (Bayley et al., 2005b; Gold and Squire, 2005). The damage in the previous studies resulted from a variety of etiologies and reportedly included diencephalic (Nichols et al., 2006) and medial temporal lobe structures (Nichols et al., 2006; Olson et al., 2006a,b). Descriptions of the damage were based on visual inspection of MR images, or on etiology in the absence of MRI evidence. In the absence of quantitative measurements, the possibility remains that the patients had additional damage.

It is also possible that differences in testing procedure or in the construction of test stimuli could account for the modest differences between our study and the previous ones. First, the faces in Olson et al. (2006b) were presented without hair, which can make face recognition rather difficult. Our faces were presented with hair (as in Nichols et al., 2006). In addition, in Olson et al. (2006a), there was not a subject-paced pause between trials (instead, there was a $0.5 \mathrm{~s}$ intertrial interval). In our experience, amnesic patients can become confused about what they are supposed to do or whether they are in the study phase or the test phase. Therefore, we included a pause between trials, so that the patients would not be disadvantaged in their knowledge about the task compared with controls. This difference in procedure might explain why our patients performed a little better than the patients in Olson et al. (2006a).

Using a measure of working memory that is unrelated to the effects of medial temporal lobe damage, we have resolved a circularity inherent in the working memory construct. The findings support a brain-based distinction between working memory and long-term memory, as well as the idea that working memory is independent of medial temporal lobe structures. We suggest that working memory depends on persistent activity in distributed regions of neocortex, including frontal, lateral temporal, and parietal cortical areas that are known to be important in the perception and initial processing of new information (Fuster, 2003; Postle, 2006).

\section{References}

Atkinson RC, Shiffrin RM (1968) Human memory: a proposed system and its control processes. In: The psychology of learning and motivation: advances in research and theory (Spence KW, Spence JT, eds), pp 89-195. New York: Academic.

Baddeley A (2003) Working memory: looking back and looking forward. Nat Rev Neurosci 4:829-839.

Baddeley A, Hitch GJ (1974) Working memory. In: Recent advances in learning and motivation (Bower GA, ed), pp 47-90. New York: Academic.

Baddeley AD, Warrington EK (1970) Amnesia and the distinction between long- and short-term memory. J Verbal Learn Verbal Behav 9:176-189.

Bayley PJ, Frascino JC, Squire LR (2005a) Robust habit learning in the absence of awareness and independent of the medial temporal lobe. Nature 436:550-553.

Bayley PJ, Gold JJ, Hopkins RO, Squire LR (2005b) The neuroanatomy of remote memory. Neuron 46:799-810.

Bayley PJ, Hopkins RO, Squire LR (2006) The fate of old memories after medial temporal lobe damage. J Neurosci 26:13311-13317.

Cohen NJ, Ryan J, Hunt C, Romine L, Wszalek T, Nash C (1999) Hippocampal system and declarative (relational) memory: summarizing the data from functional neuroimaging studies. Hippocampus 9:83-98.

Drachman DA, Arbit J (1966) Memory and the hippocampal complex. II. Is memory a multiple process? Arch Neurol 15:52-61.

Ezzyat Y, Olson IR (2008) The medial trmporal lobe and visual working memory: comparisons across tasks, delays, and visual similarity. Cogn Affect Behav Neurosci 8:32-40.

Fuster JM (2003) Cortex and mind. New York: Oxford UP.

Gold JJ, Squire LR (2005) Quantifying medial temporal lobe damage in memory-impaired patients. Hippocampus 15:79-85.

Hannula DE, Tranel D, Cohen NJ (2006) The long and the short of it: relational memory impairments in amnesia, even at short lags. J Neurosci 26:8352-8359.

Hartley T, Bird CM, Chan D, Cipolotti L, Husain M, Vargha-Khadem F, Burgess N (2007) The hippocampus is required for short-term topographical memory in humans. Hippocampus 17:34-48.

Milner B (1972) Disorders of learning and memory after temporal lobe lesions in man. Clin Neurosurg 19:421-446.

Nichols EA, Kao YC, Verfaellie M, Gabrieli JD (2006) Working memory and long-term memory for faces: evidence from fMRI and global amnesia for involvement of the medial temporal lobes. Hippocampus 16:604-616.

Olson IR, Page K, Moore KS, Chatterjee A, Verfaellie M (2006a) Working memory for conjunctions relies on the medial temporal lobe. J Neurosci 26:4596-4601.

Olson IR, Moore KS, Stark M, Chatterjee A (2006b) Visual working memory is impaired when the medial temporal lobe is damaged. J Cogn Neurosci 18:1087-1097.

Pashler H, Carrier M (1996) Structures, processes, and the flow of information. In: Memory: handbook of perception and cognition (Bjork E, Bjork R, eds), pp 3-29. San Diego: Academic.

Postle BR (2006) Working memory as an emergent property of the mind and brain. Neuroscience 139:23-38.

Shrager Y, Gold JJ, Hopkins RO, Squire LR (2006) Intact visual perception in memory-impaired patients with medial temporal lobe lesions. J Neurosci 26:2235-2240.

Snodgrass JG, Vanderwart M (1980) A standardized set of 260 pictures: norms for name agreement, image agreement, familiarity, and visual complexity. J Exp Psychol [Hum Learn] 6:174-215.

Warrington EK, Taylor AM (1973) Immediate memory for faces: long- or short-term memory? Q J Exp Psychol 25:316-322.

Waugh NC, Norman DA (1965) Primary memory. Psychol Rev 72:89-104. 